\title{
Hypsikrateia / Hypsikrates: Travestie aus Liebe. König Mithradates Eupators Page und eine neue griechische Inschrift aus Phanagoreia/Rußland
}

\author{
(vorgetragen in der Plenarsitzung am 17. Juni 2011) \\ HEINZ HEINEN
}

Für Clemens Zintzen

in dankbarer und herzlicher Verbundenheit

\section{Hypsikrateia in der literarischen Überlieferung}

In seiner Sammlung denkwürdiger Taten und Worte (Facta et Dicta Memorabilia) widmet der römische Schriftsteller Valerius Maximus, ein Zeitgenosse des Kaisers Tiberius, das 6. Kapitel von Buch IV der Gattenliebe. ${ }^{1}$ Unter dem Titel de amore coningali stellt der Autor seinen Lesern Bilder legitimer Ehen vor Augen, die höchster Verehrung würdig sind. Seiner üblichen Anordnung folgend bietet Valerius Maximus zunächst einige römische Exempla, um dann anschließend Beispiele der Gattenliebe bei anderen Völkern anzuführen. ${ }^{2}$ Den Anfang macht Königin Artemisia, Gattin des Königs Mausolos von Karien im südwestlichen Kleinasien.

Als zweites Beispiel ausländischer Gattenliebe folgt eine weitere asiatische Königin, Hypsicratea.

Val. Max. IV 6 ext. 2: De amore coniugali externorum

Hypsicratea quoque regina Mithridatem coningem suum effusis caritatis habenis amauit, propter quem praecipuum formae suae decorem in habitum uirilem conuertere uoluptatis loco habuit: tonsis enim capillis equo se et armis adsuefecit, quo facilius laboribus et pericu-

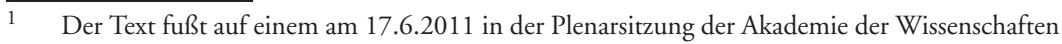
zu Göttingen gehaltenen Vortrag. Für die Veröffentlichung ist er erweitert und um Anmerkungen ergänzt worden. Frühere Fassungen konnte ich am 4.10.2010 in Zürich und am 3.2.2011 in Trier vorstellen. Den Teilnehmern dieser Veranstaltungen danke ich für manche Anregung, zusätzlich Andrea Binsfeld, Sabine Heck, Hans-Otto Kröner und Georg Wöhrle für die kritische Durchsicht einer ersten Version des Textes, sowie Luis Ballesteros Pastor und Oleg L. Gabelko für freundlich gewährte Einsicht in noch ungedruckte Aufsätze. Für die redaktionelle Gestaltung bedanke ich mich ganz besonders bei Johannes Deißler, Akademie der Wissenschaften und der Literatur, Mainz.

2 Zum Werk des Valerius Maximus und zu seiner Person vgl. die ausführliche Einleitung von R. Combès in seiner Ausgabe: Valère Maxime 1995, S. 7-62, sowie die Einleitung in ThemannSteinke 2008, S. 16-47, dort S. 17-28 zu den Kontroversen um die Datierung des Valerius Maximus. Zu seinen Quellen s. auch Bosch 1929 und Klotz 1942, zum Aufbau seiner ExemplaSammlung Thurn 2001, zu den fremden Frauen in seinem Werk Ballesteros Pastor 2004. 
lis eius interesset. quin etiam uictum a Cn. Pompeio per efferatas gentes fugientem animo pariter et corpore infatigabili secuta est. cuius tanta fides asperarum atque difficilium rerum Mithridati maximum solacium et iucundissimum lenimentum fuit: cum domo enim et penatibus nagari se credidit, uxore simul exulante.

„Auch die Königin Hypsicratea ließ in der Liebe zu ihrem Gatten Mithridates die Zügel schießen. Denn es war ihr ein Vergnügen, für ihn den Hauptschmuck ihrer Schönheit in eine männliche Tracht zu verwandeln. Denn die Haare kurz geschnitten, gewöhnte sie sich an Pferd und Waffen, um desto leichter seine Mühen und Gefahren zu teilen. Sogar nachdem er von Cn. Pompeius besiegt worden war und seine Flucht ihn durch (die Gebiete) wilder Völker führte, folgte sie ihm, an Mut und Körper gleichermaßen unermüdlich. Eine solche Treue in bitterer und schwerer Lage war für Mithridates größter Trost und angenehmste Linderung, glaubte er doch mit Haus und Penaten zu ziehen, da seine Frau den Verlust der Heimat mit ihm teilte." (Übers.: H. Heinen)

Der Passus enthält eine Reihe bemerkenswerter Nachrichten. Er beginnt mit der Verwandlung der Hypsicratea, die ihr langes Haar opfert und männliche Tracht anlegt, um als Page dem von ihr geliebten Mann, König Mithradates Eupator von Pontos in Kleinasien, ganz nahe sein zu können. Ihre außerordentliche Treue (fides) beweist sie in schwierigster Lage, auf der Flucht des Mithradates nach der verlorenen Schlacht gegen Pompeius.

Wie so oft hat Valerius Maximus auch dieses Exemplum als Einzelbild aus seinem Kontext herausgelöst. Doch ehe wir den historischen $\mathrm{Zu}$ sammenhang rekonstruieren, wollen wir uns noch ein weiteres Zeugnis zu Hypsicratea, griech. Hypsikrateia, vor Augen führen. ${ }^{3}$ Es steht in der Pompeius-Vita Plutarchs (32, 13-15) und liefert uns weitere wertvolle Anhaltspunkte im Rahmen seines entscheidenden Sieges über Mithradates.

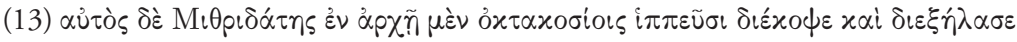

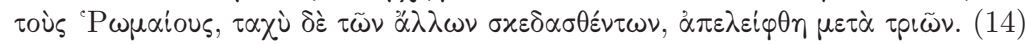

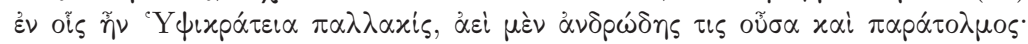

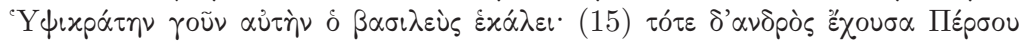

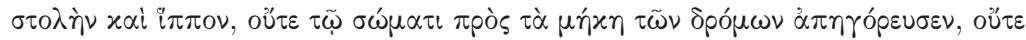

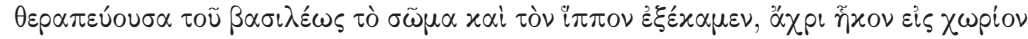

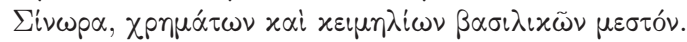

„(13) Mithridates selbst sprengte im Anfang an der Spitze von achthundert Reitern die Römer auseinander und schlug sich durch, dann aber hatten sich schnell die anderen alle verlaufen, und er war mit dreien allein gelassen, (14) unter denen sich seine Nebenfrau Hypsikrateia befand, die stets männliche Verwegenheit bewies; Hypsikrates nannte sie daher der König. (15) Sie trug damals die Kleidung eines Persers, saß zu Pferde, versagte körperlich nicht trotz der Länge der Ritte und wurde nicht müde,

3 Im Folgenden verwende ich durchgehend die griechischen Namensformen Hypsikrateia und Mithradates, im letzteren Falle den offiziellen Inschriften folgend. 
den Leib des Königs und sein Roß zu versorgen, bis sie zu der Burg Sinora kamen, die mit königlichen Schätzen und Kleinodien angefüllt war." (Übers.: K. Ziegler) ${ }^{4}$

Im Vergleich mit Valerius Maximus stellt Plutarch die Figur Hypsikrateias deutlich distanzierter dar. ${ }^{5}$ Von ihm hören wir, dass sie eine pallakis, eine Nebenfrau bzw. Konkubine, des pontischen Königs gewesen sei. Hier wird sie also, im Gegensatz zu Valerius Maximus, nicht als uxor oder coniunx, folglich nicht als Musterbeispiel eines legitimus amor, vorgestellt. Diesen Unterschied wollen wir mit Blick auf die Weiterentwicklung unseres Themas im Auge behalten. Bemerkenswert ist weiterhin, dass der König sie wegen ihres männlichen Aussehens und Auftretens mit der maskulinen Form ihres Namens, Hypsikrates statt Hypsikrateia, anredete. Ergänzend zu den Hinweisen des Valerius Maximus über die Tracht der Hypsikrateia erfahren wir von Plutarch, dass sie persische Männerkleidung trug. Eine junge Frau mit kurzen Haaren und in Männertracht, eine verwegene und ausdauernde Reiterin, so steht sie vor uns, Hypsikrateia, der Page des Königs Mithradates Eupator. Bei diesem Bild muss man unwillkürlich an Gustav Adolfs Pagen Leubelfing, die tragische Heldin von Conrad Ferdinand Meyers bekannter Novelle, denken, an Gustel Leubelfing, die der Dichter mit folgenden Worten einführt: „ein tannenschlankes Mädchen mit lustigen Augen, kurzgeschnittenen Haaren, knabenhaften Formen und ziemlich reitermäßigen Manieren"6.

Ehe wir uns durch diesen Vergleich zu weiteren Überlegungen anregen lassen, wollen wir zuvor den historischen und geographischen Zusammenhang rekonstruieren, in den wir die Auszüge aus Valerius Maximus und Plutarch einfügen müssen. Den Kontext bietet Plutarch: Die letzte große Schlacht des Mithradates gegen den römischen Feldherrn Pompeius fand in Kleinarmenien, im nordöstlichen Kleinasien, statt. Die Truppen des pontischen Königs wurden eingekesselt und vernichtend geschlagen. Weit mehr als zehntausend seiner Leute fanden den Tod. Mithradates selber entkam und setzte seine Flucht mit nur dreien seiner Getreuen, darunter Hypsikrateia, fort. Ganz besonders hebt Plutarch hervor, dass der Kampf in der Nacht stattfand, auf einem vom untergehenden Mond noch schwach be-

\footnotetext{
4 In: Plutarch 1955, S. 195.

5 Während meine Ausführungen deutlich auf Valerius Maximus ausgerichtet sind (Quellen seiner Darstellung und vor allem Travestie der Hypsikrateia), konzentriert Ballesteros Pastor 2011 sich stärker auf die Darstellung Plutarchs. Er betont (S. 114ff.) dessen kritische Beurteilung Hypsikrateias und deutet dies als einen weiteren Versuch des Biographen, aus einer perserfeindlichen Perspektive Mithradates als orientalischen Herrscher zu charakterisieren und zu diskreditieren.

6 Meyer 1959, S. 170. Die Novelle erschien zuerst 1882 in der Monatsschrift „Deutsche Rundschau" unter dem Titel Page Leubelfing, bald darauf in Buchform unter dem Titel Gustav Adolfs Page.
} 


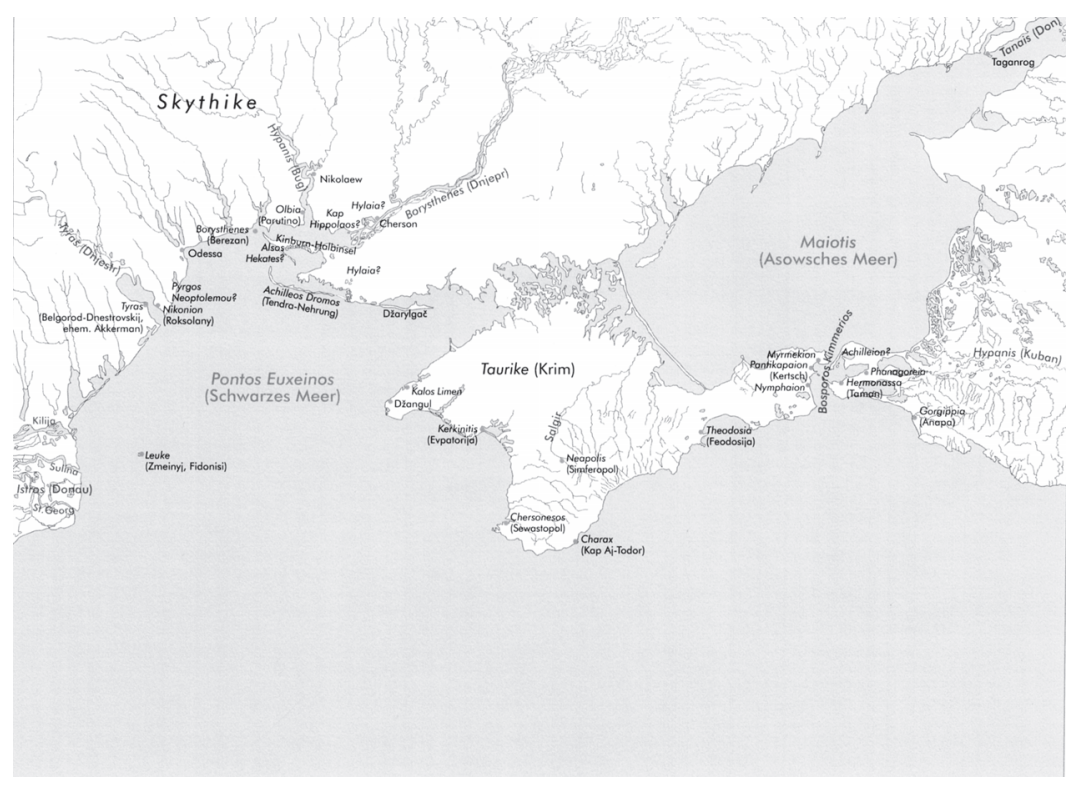

Abbildung 1: Der nördliche Schwarzmeerraum

schienenen Kampfplatz. ${ }^{7}$ Wir sind im Jahre 65, kurz vor der Endphase des langen Krieges zwischen Mithradates und den Römern. Nach der Niederlage flieht der König weiter nach Norden, über Kolchis am Ostufer des Schwarzen Meeres. Sein Ziel ist Pantikapaion, seine Residenz an der Ostküste der Krim (Karte Abbildung 1). Doch der Aufstand seines eigenen Sohnes Pharnakes treibt den König 63 v. Chr. in den Tod, nachdem er noch einmal versucht hatte, neue Kräfte gegen die Römer zu mobilisieren.

\section{Eine neue Entdeckung: das Grabdenkmal Hypsikrateias}

In den detailreichen Berichten über den Untergang des Mithradates, über die Tapferkeit seiner Töchter und den Verrat seines Sohnes Pharnakes spielt Hypsikrateia erstaunlicher Weise keine Rolle, obwohl man die treue Begleiterin und Mitkämpferin des Königs gerade unter diesen dramatischen Umständen an seiner Seite erwartet hätte. Die antiken Autoren scheinen Hypsi-

7 Plut. Pomp. 32, 10-12. Vgl. Reinach 1895, S. 384f. Die in Teilen der antiken Überlieferung malerisch ausgestalteten Details dieser nächtlichen Schlacht offenbaren Widersprüche und sind in der modernen Forschung häufig bestritten worden. Vgl. z.B. Heftner 1995, S. 61f. und 227ff., sowie Ballesteros Pastor 1997, S. 245. 
krateia gewissermaßen aus den Augen verloren zu haben. Doch im Jahre 2005 ist sie unerwartet wieder aufgetaucht, im buchstäblichen Sinne wieder aufgetaucht, denn ein Teil ihres Grabdenkmals wurde bei Unterwassergrabungen in der Straße von Kertsch, dem Kimmerischen Bosporos, entdeckt. Der Fundort liegt im versunkenen Teil der Griechenstadt Phanagoreia, ${ }^{8}$ vor der Küste der Halbinsel Taman, gegenüber der Ostküste der Krim und Pantikapaion. An dieser Stelle haben die Forscher des Archäologischen Instituts der Russländischen Akademie der Wissenschaften in den Kampagnen der Jahre 2004 und 2005 u. a. vier Inschriftenblöcke gehoben, ${ }^{9}$ darunter (2005) einen Statuensockel, dessen Oberseite für die Aufstellung einer Statue hergerichtet war. Die aus Marmor gefertigte Basis ist ganz offenkundig von ihrem ursprünglichen Platz entfernt und in der Hafenanlage von Phanagoreia verbaut worden. Auf der Vorderseite der Basis befindet sich ein höchst erstaunlicher Text (Abbildung 2 und 3). ${ }^{10}$

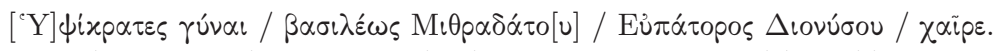
„Hypsikrates, Frau des Königs Mithradates Eupator Dionysos, lebe wohl!“

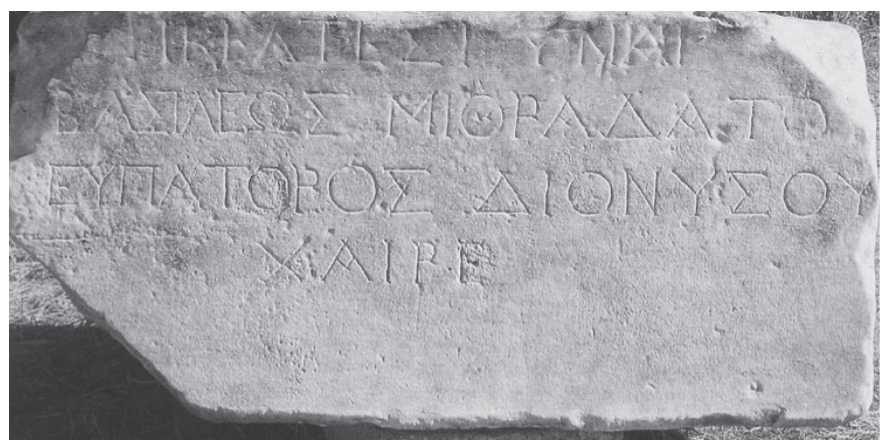

Abbildung 2: Statuenbasis mit Grabinschrift der Hypsikrateia/Hypsikrates

$8 \quad$ Zur Orientierung über Phanagoreia vgl. Kuznetsov/Povalahev 2009.

9 Russische ed. princ. der vier Inschriften: Kuznecov 2006 und Kuznecov 2007; französische Edition: Bongard-Levine u.a. 2006; vgl. SEG 56 (2006 [erschienen 2010]) Nr. 931-934. Zur Auswertung s. auch Heinen 2008.

10 Französische ed. princ.: Bongard-Levine u.a. 2006, S. 277f. (nicht fehlerfrei), Foto des Denkmals in situ ebd. S. 279, Abb. 14. Maßgeblich ist die russische ed. princ.: Kuznecov 2007 (1) S. 238-243; Foto der Statuenbasis (von oben) ebd. Tafel nach S. 128 Abb. 5, Foto der Inschriftseite ebd. Abb. 6. Die Statuenbasis ist beschädigt; ihre Maße: $85-88$ x 76,9-79 cm, Höhe 41,5 cm. Wiedergabe und Diskussion der Inschrift: SEG 56 (2006) Nr. 934, und Bull. ép. 2008, Nr. 432 (A. Avram); wichtige und weiterführende Beobachtungen zur Inschrift und zur Person Hypsikrateias von P. Bernard apud Bongard-Levine u.a. 2006, S. 279-288; Bowersock 2008, S. 600f.; Gabelko 2009 (dort S. 188f. Korrekturen zur französischen ed. princ.), sowie Ballesteros Pastor 2011. 


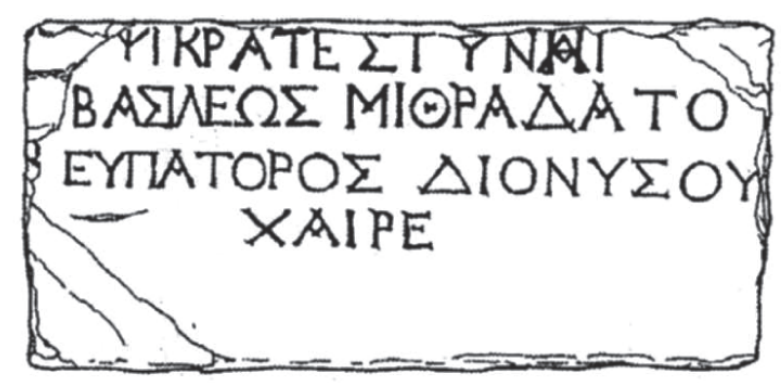

Abbildung 3: Umzeichnung von Abbildung 2

Der Wortlaut lässt keinen Zweifel: Wir haben es wirklich mit Hypsikrateia, Page und Gefährtin des Mithradates, zu tun. Der König trägt in dieser Inschrift seinen dynastischen Beinamen Eupator, der seine vornehme Abstammung zum Ausdruck bringt. Überdies lesen wir hier den kultischen Beinamen Dionysos, denn als ein neuer Dionysos trat Mithradates auf, um wie ein Heil und Segen bringender Gott seinen machtpolitischen Anspruch in eine religiöse Form zu kleiden und im Kampf gegen seine kleinasiatischen Rivalen und gegen Rom zu verkünden. ${ }^{11}$ Halten wir des Weitern fest, dass Hypsikrateia in der neuen Inschrift als Frau (gyné) des Königs erscheint, im Gegensatz zu ihrer Bezeichnung als pallakis bei Plutarch und ganz im Einklang mit Hypsikrateias Stellung als coniunx bzw. uxor im Wortlaut des Valerius Maximus. Das Erstaunlichste an diesem Text ist jedoch die Bezeichnung Hypsikrateias als Hypsikrates, und zwar in der Vokativform, passend zum Imperativ chaîre, „Lebe wohl!“, dem typischen Abschiedsgruß auf griechischen Grabdenkmälern. Die bisweilen ungläubig registrierte Aussage in dem oben zitierten Passus Plutarchs, der König habe seinen Pagen mit der männlichen Namensform angesprochen (Pomp. 32, 14), findet auf diesem aus dem Meer geborgenen Denkmal ihre überraschende und unwiderlegbare Bestätigung. Nach der Beschaffenheit des Sockels zu urteilen, stand dieses Denkmal nicht allein, sondern war Teil einer größeren Anlage, vielleicht - so die Vermutung des Ausgräbers V.D. Kuznecov - eines Grabmonuments für mehrere beim Aufstand des Jahres 63 ums Leben gekommene Anhänger des Mithradates. ${ }^{12}$ Gerade im Falle eines Denkmals für mehrere Personen könnte es sich um ein kommemoratives Monument handeln, ohne dass sicher wäre, dass die sterblichen Überreste der Betreffenden sich wirklich in der Anlage befanden. Ange-

11 Vgl. Bohm 1989, S. 153-191, zur Propaganda des Mithradates.

12 Kuznecov 2007, S. $242 \mathrm{f}$. 
sichts der turbulenten militärischen Ereignisse des Jahres 63 in Phanagoreia sollte man deshalb die Möglichkeit eines Kenotaphs nicht ausschließen und bei der Bezeichnung, Grabdenkmal' mit in Betracht ziehen. Hoffentlich bringen weitere Entdeckungen und Untersuchungen genaueren Aufschluss über Konstruktion, Intention und Chronologie dieses bemerkenswerten Ensembles.

Ein solcher Fund ist ein extraordinärer Glücksfall der Archäologie und im wahrsten Sinne ein kleines Wunder. Und wie so manches Wunder wird auch der Fund der russischen Archäologen angezweifelt. Der ungläubige Thomas heißt in diesem Falle Filippo Canali De Rossi, ein kenntnisreicher italienischer Historiker und erfahrener Epigraphiker. In der bekannten amerikanischen Internetrezensionszeitschrift Bryn Mawr Classical Review (2009.05.22) schreibt er (Anm. 7): „A mio modesto parere la perfetta rispondenza del testo epigrafico alla notizia tramandata da Plut. Pomp. 32, 13-16 potrebbe far insorgere qualche dubbio sulla effettiva autenticità dell'iscrizione.“ - „Nach meinem bescheidenen Dafürhalten könnte die perfekte Entsprechung zwischen dem epigraphischen Text und der von Plut. Pomp. 32, 13-16 überlieferten Notiz gewisse Zweifel an der tatsächlichen Echtheit der Inschrift hervorrufen." - Zwar hüllen sich die Zweifel des italienischen Kollegen in den Mantel der Bescheidenheit und in die Form des Konditionalis, doch ist es ziemlich unerhört, eine derart gut dokumentierte archäologische Operation in dieser Weise zu verdächtigen. ${ }^{13}$ Die bruchstückhafte Statuenbasis und die Ausführung der Inschrift sprechen nicht gerade für eine Fälschung. Ein Blick auf die erste Zeile des Textes zeigt, dass der Steinmetz einen Irrtum begangen und zunächst die Nominativform gyné, „Frau“, eingemeißelt hatte. Dann korrigierte er seinen Fehler und setzte richtiger Weise die Vokativform gýnai ein, passend zum Vokativ Hypsikrates und zum Imperativ chaîre, „Lebe wohl!“. Es besteht, nicht nur meiner Meinung nach, wirklich kein Grund, an der Echtheit der Inschrift zu zweifeln. Der von Canali De Rossi geäußerte Verdacht zeigt gewissermaßen e negativo, mit welcher außerordentlichen, fast (aber eben nur fast) unglaublichen Entdeckung wir es hier zu tun haben. ${ }^{14}$

Leider ist die Statue der Hypsikrateia, die ursprünglich auf dem Sockel dieses Denkmals stand, verloren oder, optimistischer, noch nicht wieder gefunden. Wie dürfen wir uns die verstorbene Gattin des Mithradates auf

\footnotetext{
13 Vgl. nur das Foto des Denkmals in situ: Bongard-Levine u.a. 2006, S. 279, Abb. 14.

14 Auf die überaus phantasievollen Spekulationen von Mayor 2011, S. 415-417 (engl.: Mayor 2010, S. 365-369) hinsichtlich der Inschrift und vor allem des Überlebens der Hypsikrateia unter dem Namen und in der Person des Gelehrten Hypsikrates (zu ihm vgl. F. Jacoby. RE IX 1 [1914] Sp. 433 f., s.v. Hypsikrates, 1) muss ich nicht näher eingehen.
} 
dieser Statuenbasis vorstellen? Dass sogar für dieses offizielle Monument die männliche Namensform Hypsikrates statt Hypsikrateia gewählt wurde, zeigt, dass diese Anredeform kein Scherz des Mithradates war, wie man früher angenommen hatte, ${ }^{15}$ sondern fest mit seiner Trägerin verbunden war. Daraus dürfen wir schließen, dass die Verstorbene auf diesem Denkmal in ihrer männlichen Erscheinungsform verewigt wurde, d.h. mit kurzen Haaren und mit dem Hosengewand (anaxyrídes), das für iranische Krieger seit alters und auch damals üblich war. ${ }^{16}$

\section{Thematische Schwerpunktsetzung}

Die neue Inschrift wirft in Verbindung mit den eingangs zitierten literarischen Zeugnissen eine ganze Reihe von Fragen auf, denen hier, allein schon aus Platzgründen, auch nicht annähernd vollständig nachgegangen werden kann, zumal inzwischen bereits mehrere einschlägige Untersuchungen vorliegen. ${ }^{17}$ Bewusst klammere ich deswegen alle Fragen nach dem Status Hypsikrateias (Konkubine, Ehefrau, Königin?) aus. ${ }^{18}$ Die neue Inschrift nennt sie gyné. Dem entspricht bei Valerius Maximus ihre Nennung als uxor ihres Ehemannes (coniunx) Mithradates und ihre Aufnahme unter die Exempla für amor coniugalis. Valerius Maximus nennt sie zudem regina. Diesen königlichen Rang nennt ihre Inschrift jedoch nicht. Deren erste Zeile weist mit der ursprünglichen Verwechslung des Nominativs und des Vokativs von gyné allerdings Besonderheiten auf, die zu Vermutungen Anlass gegeben haben, auf die ich hier nicht näher eingehe. ${ }^{19}$ Die Texte zu Hypsikrateia bieten sich für Fragen der Genderforschung geradezu an, doch möchte ich auch diese Fragestellung im vorliegenden Beitrag nicht eingehender behandeln, sondern mich auf einen bestimmten Aspekt konzentrieren: die Travestie Hypsikrateias. Ihre Übernahme eines habitus virilis - so Valerius Maximus in dem eingangs zitierten Text - ist keine Verkleidung mit Täuschungsabsicht und trägt offenkundig auch keine pathologischen Züge, wie sie bei Transvestiten oder im Umfeld von cross-gender und cross-

15 Reinach 292: „... Hypsikrateia, eine kühne Reiterin von männlichem Gebahren (sic), weswegen sie der König scherzweise Hypsikrates zu nennen pflegte."

16 Vor allem seit den Perserkriegen wurden des Öfteren auch die Amazonen mit einem solchen Hosengewand dargestellt. Vgl. etwa die Abbildungen in: Historisches Museum der Pfalz Speyer 2010, S. 94f. Hier zeigt sich der steppennomadische, skythische, Einfluss auf das Bild der Amazonen.

17 S. die in Anm. 10 zitierte Literatur.

18 Vgl. dazu Ballesteros Pastor 1997, S. 245; Gabelko 2009, S. 197-199, u. a. mit dem Hinweis auf einen einschlägigen Aufsatz von K.L. Gulenko (non vidi).

19 Vgl. die Überlegungen von Gabelko 2009, S. 193-201. 
dressing vorkommen können. Ohne leugnen zu wollen, dass das Verhalten Hypsikrateias in Wirklichkeit komplexer sein könnte und andere Erklärungsmuster ebenfalls gültig sein mögen, versuche ich ihr Verhalten und ihr Erscheinungsbild als Travestie aus Liebe zu deuten und in den Kontext ähnlicher Fälle zu stellen. Auch diese Parallelen weisen jeweils eigene Züge auf und stellen ohnehin nur eine ganz kleine Auswahl dar. Dabei ist klar, dass Hypsikrateias Travestie aus Liebe in einem für uns nicht mehr durchschaubaren Maße eine Konstruktion ist, die bereits in den Quellen des Valerius Maximus vorgenommen worden war und bei Letzterem durch die Einfügung in das Exempla-Schema sicherlich noch verstärkt worden ist. Das neu entdeckte Denkmal Hypsikrateias in Phanagoreia sollte jedoch davor warnen, die Dekonstruktion zu weit zu treiben.

\section{Von Amazonen und iranischen Reiterkriegerinnen}

Wir wissen nicht, woher Hypsikrateia stammte, am ehesten wohl aus Kleinasien, vielleicht aus dem ursprünglichen Reichsgebiet des Königs Mithradates im nordöstlichen Kleinasien. Das wäre nicht weit von der Heimat der mythischen Amazonen. Es fällt jedoch auf, dass sowohl Valerius Maximus als auch Plutarch der doch sehr nahe liegenden Versuchung widerstehen, Hypsikrateia als eine Art zeitgenössische Amazone zu bezeichnen und darzustellen. Dennoch ist es sicher legitim, in diesem Zusammenhang an die Tradition der Amazonen zu erinnern. ${ }^{20}$ Doch die wirklichen Parallelen für eine Gestalt wie Hypsikrateia sollten wir nicht so sehr im Mythos, sondern eher im Kontext der tatsächlich nachgewiesenen Reiterkriegerinnen der östlichen Steppen, etwa bei den iranischen Skythen und Sarmaten des nördlichen Schwarzmeerraums, suchen. ${ }^{21}$ Auch im pontischen Reich des

$20 \quad$ Diesen Aspekt vor allem betont Ballesteros Pastor 1997 mit interessanten Beobachtungen zu Hypsikrateia. Wie verführerisch, gerade für die Propaganda des Pompeius, die Assoziierung seines Kaukasusfeldzuges mit den legendären Amazonen sein konnte, zeigen u. a. folgende Belege: Plut. Pomp. 35 berichtet von einem Sieg des Pompeius über eine einheimische Streitmacht im Kaukasus. Auf dem Schlachtfeld seien Amazonenwaffen, aber keine Leichen von Amazonen gefunden worden $(35,5)$. Vgl. Strab. 11, 5 (503f.) zu den Amazonen im weiteren Zusammenhang des Kaukasusfeldzugs. Zu den im Triumphzug des Pompeius mitgeführten und als Amazonen betrachteten Frauen s. App. Mithr. 482f. (vgl. Plut. Pomp. 45, 5), und Östenberg 2009, S. 148 (teilweise spekulativ).

21 Eine Leiche mit Waffenbeigabe und typischem Frauenschmuck befand sich zum Beispiel in einem sarmatischen Familiengrab des 4. Jh. v. Chr. (Kurgan Nr. 4 beim chutor [Anwesen] Sladkovskij, Gebiet Rostov am Don): Smirnov 1982. Zahlreiche weitere Funde und Diskussionen in: Historisches Museum der Pfalz Speyer 2010. Zur weiteren Orientierung siehe Fornasier 2007. Plat. Nom. 804e-805a nennt die zahlreichen berittenen und bewaffneten Sauromatinnen am Schwarzen Meer und vergleicht sie 806a - differenzierend - mit den Amazonen. - Die 
Mithradates lebten iranische Traditionen weiter. Der König berief sich voller Stolz nicht nur auf seine makedonisch-hellenistischen Ahnen, sondern auch auf seine persischen Vorfahren aus dem Haus der Achämeniden. ${ }^{22}$ Im Umkreis dieser iranischen Reitereliten dürfen wir das Vorbild der Hypsikrateia suchen, deren persische Tracht Plutarch in dem zitierten Passus ausdrücklich hervorhebt. ${ }^{23}$ Dennoch war Hypsikrateia offenbar keine alltägliche Erscheinung in dem durch viele unterschiedliche Traditionen geprägten Umfeld des Mithradates. Vor allem: Im Gegensatz zur Männerfeindlichkeit der legendären Amazonen hatte sie ihre Angleichung an den habitus virilis aus Liebe zu einem Mann, König Mithradates, vollzogen.

Demzufolge ist die Gefährtin des Mithradates einer in Anatolien noch sehr lebendigen persisch-iranischen Tradition und einem kriegerischen Frauentyp zuordnen, der sowohl durch die Amazonen des Mythos als auch die real kämpfenden Frauen der osteuropäischen Reiternomaden repräsentiert wird. Typologisch verwandt in einem weiteren Sinne sind natürlich jene Mädchen und Frauen aller Zeiten, die aus den verschiedensten Beweggründen in die Männerdomäne des Krieges eingedrungen sind und sich in Habitus und Tracht ihrer männlichen Umwelt angepasst haben. Dazu gehören historische Gestalten wie Jeanne d'Arc oder lebensechte Kriegerinnen in Liedern und Erzählungen, in Romanen und Filmen. ${ }^{24}$ Dazu zählt auch Gustav Adolfs vorhin erwähnter Page Gustl Leubelfing, eine reizvolle Phantasiefigur Conrad Ferdinand Meyers, die sich den Herzenswunsch erfüllt, an der Seite des von ihr verehrten Schwedenkönigs zu leben und zu kämpfen. Allerdings muss sie ihre Mädchennatur geheim halten. Eine Frau als Page des streng protestantischen Gustav Adolf war in der Wirklichkeit undenkbar. Es bedurfte der Tarnung und Verstellung der Gustl Leubelfing, um ihr Ziel dennoch zu erreichen. ${ }^{25}$ An der Seite des Mithradates und im Umfeld seines ,Harems ' war im Falle Hypsikrateias keine solche Ge-

in den Jahren 1983 und 1990 auf der Tamanhalbinsel, also in der weiteren Region von Phanagoreia, entdeckten Fragmente eines Kampfreliefs spätklassischer oder hellenistischer Zeitstellung sind keinesfalls sicher als Amazonomachie zu deuten. Vgl. dazu die unterschiedlichen Standpunkte in dem Sammelband von Savostina 2001.

22 Iust. 38, 7, 1. Zur Selbstdarstellung und Propaganda des Mithradates vgl. Bohm 1989, S. 153 191, und Ballesteros Pastor 1996, S. 379-416.

23 Ballesteros Pastor 2011, S. 114f. stellt den Hinweis auf die persische Tracht Hypsikrateias in den Rahmen der negativen Charakterisierung des Mithradates als eines orientalischen Herrschers durch Plutarch.

24 Die umfangreiche Literatur kann hier nicht angeführt werden. Vgl. z.B. nur Seemann 1959 zu den Kriegerinnen in den europäischen Volksballaden und Altenburger 2009 zur Gestalt des weiblichen Ritters in der chinesischen Tradition.

25 Zu den für C. F. Meyer wichtigen Themen Maske und Identität s. Bang 1940 und Wiesmann 1958. 
heimhaltung vonnöten. ${ }^{26}$ Gleichwohl schlüpfte auch sie in eine Rolle, die für Frauen ihrer Zeit ungewöhnlich war. Ihre weibliche Identität brauchte sie im Gegensatz zu Leubelfing nicht zu verbergen, denn sie gehörte zur Gruppe der Frauen und Konkubinen des Mithradates. Doch die Metamorphose Hypsikrateias und ihre äußere Umwandlung in einen Mann waren offenkundig erforderlich, um als Page an der Seite des Königs inmitten seiner Soldaten, Offiziere und des ganzen Feldlagers dienen und bestehen zu können.

Kriegerische Frauen haben in der Antike literarische Beachtung gefunden, weil sie gegen das geltende Rollenverständnis verstießen, so wie heute Frauen beim Militär, besonders im Kampfeinsatz, immer noch Aufmerksamkeit erregen und ein Thema kontroverser Debatten sind. Die antike Irritation spiegelt sich vielleicht am deutlichsten in der ambivalenten Bewertung der im Mythos beheimateten und der griechischen Kulturwelt etwas unheimlichen Amazonen. Sie stellten eine den patriarchalischen Ordnungen fremde und häufig feindliche Gegenwelt dar. ${ }^{27}$ Positiv hingegen wurden in der griechischen und der römischen Tradition jene Frauen dargestellt, die im Dienste ihres Landes oder einer gerechten Sache außerordentlichen Mut bewiesen und gelegentlich zu den Waffen gegriffen hatten. Beispiele dafür finden sich in den antiken Katalogen bedeutender Frauen, so etwa in Plutarchs Traktat über die Tüchtigkeit der Frauen (Gynaikôn aretai, Mulierum virtutes). Zwar handelt es sich hier zumeist um Frauen, die als solche, und nicht in männlicher Aufmachung, auftraten. Dennoch schlüpften einige von ihnen in Männerkleider, um eine außerordentliche Tat zu vollbringen. ${ }^{28}$ Nicht minder eindrucksvoll ist die Galerie von Frauengestalten in den Strategémata des Griechen Polyän (zweite Hälfte des 2. Jh. n. Chr.). Seine Aufzählung $(8,26-71)$ beginnt mit der babylonischen Königin Semiramis und umfasst interessanterweise auch zwei kriegerische Frauen aus dem nördlichen Schwarzmeerraum: die Mäotin Tirgatao (Strateg. 8, 55) und die Sarmatin Amage (Strateg. 8, 56). ${ }^{29}$ Eine Figur wie Hypsikrateia hätte sich vorzüglich in einen solchen Rahmen einfügen lassen, doch stellt man mit einigem Erstaunen fest, dass sie nicht nur im Werk Polyäns fehlt,

\footnotetext{
26 Zur Familie und zu den Liebesverhältnissen des Mithradates vgl. u. a. Plut. Pomp. 36, 3; Reinach 1895, S. 290-293, und vor allem Ballesteros Pastor 1996, S. 310-324 (La familia real).

27 Zur Ambivalenz der antiken und bisweilen auch modernen Vorstellungen vgl. die vielfältigen Beiträge in dem Sammelband Historisches Museum der Pfalz Speyer 2010.

28 S. die Tyrrhenerinnen in Plut. Mul. virt. 247 A-E; vgl. Polyaen. Strateg. 7, 49. Auch Val. Max. 4, 6 ext. 3 hat dieses Beispiel aufgegriffen. Gewissermaßen nur en passant weise ich im Zusammenhang von $M u l$. virt. auf Fälle von freiwilligem oder erzwungenem cross-dressing hin: 245 E-F, 247 BC und $261 \mathrm{~F}$.

29 Zu Tirgatao vgl. Gardiner-Garden 1986, S. 194-207.
} 
sondern vor allem auch in den Mulierum virtutes Plutarchs, obwohl dieser die Geschichte Hypsikrateias kannte und, wie wir vorhin gehört haben, in seiner Pompeius-Biographie erwähnte. Valerius Maximus dagegen hat Hypsikrateia in seine Exempla-Sammlung aufgenommen.

\section{Die Quelle des Valerius Maximus}

Die Quelle, aus der Valerius Maximus ihre Geschichte geschöpft hat, können wir dank der spätantiken Epitomatoren, die die Flucht des Mithradates in der berühmten nächtlichen Entscheidungsschlacht erwähnen, etwas genauer bestimmen. Besonders pathetisch ausgefallen ist trotz Kürzung der Bericht des christlichen Priesters Orosius in seinen Historiae adversum paganos (Geschichte gegen die Heiden) 6, 4, 6:

rex inter tumultus belli fuga lapsus, adiutus etiam beneficio sublustris noctis euasit, relictusque ab omnibus amicis philosophis scriptoribus rerum vel carminum ac medicis solus per deuia equum manu trahens atque ad omnes nocturnos strepitus trepidans, in quoddam castellum deuertit atque inde in Armeniam perrexit.

„Der in den Wirren des Kampfes auf der Flucht gestrauchelte König (gemeint ist Mithradates) entrann im Schutz des Halbdunkels der Nacht. Verlassen von allen Freunden, Philosophen, Prosaschriftstellern, Dichtern und Ärzten nahm er, allein sein Pferd auf Schleichwegen mit der Hand führend und bei allen nächtlichen Lauten zitternd, Zuflucht in irgendeinem Kastell und brach von dort nach Armenien auf." (Übers.: A. Lippold) ${ }^{30}$

In der dramatisierenden Zuspitzung dieses Fluchtberichtes befindet sich Mithradates ganz allein und von allen verlassen. Orosius' Darstellung fußt in diesen Partien auf dem Geschichtswerk Ab urbe condita des Livius. Müssen wir nun aus dem Fehlen Hypsikrateias im Resümee des Orosius schließen, dass auch Livius von der treuen Begleiterin des Mithradates nichts wusste? Allein schon aus methodischen Gründen dürfen wir in diesem Falle kein argumentum e silentio zulassen, denn in der Volldarstellung des Livius kann, ja muss vieles gestanden haben, was in der Kurzfassung des Orosius fehlt. Dass es sich tatsächlich so verhält, lehrt ein Blick auf das gleichfalls spätrömische Breviarium des Eutrop, der sich wie Orosius für die Geschichte der römischen Republik auf Livius stützte. Er berichtet, dass Mithradates mit seiner Frau und zwei Begleitern aus der Schlacht floh: Mithridates cum uxore fugit et duobus comitibus $(6,12)$. Damit dürfte nun klar sein, dass

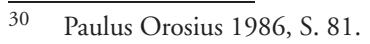


auch in der Darstellung des Livius die Flucht des Mithradates in Begleitung seiner Frau nicht fehlte. ${ }^{31}$

Wir können nur vermuten, aber nicht beweisen, dass Livius den Namen Hypsikrateias nicht nur kannte, sondern im Zusammenhang der Flucht auch genannt hat. Vieles spricht dafür, dass Livius tatsächlich die Quelle der so anschaulichen Präsentation der Hypsikrateia bei Valerius Maximus gewesen ist, zumal wir wissen, dass Valerius Maximus auch sonst häufig aus Livius geschöpft hat. So könnte es ebenfalls im Falle Hypsikrateias gewesen sein, vor allem da auch der auf Livius fußende Eutrop, wie Valerius Maximus, die Begleiterin des Mithradates als uxor bezeichnet.

\section{Die Niederlage des Mithradates und der Triumphzug des Pompeius (61 v. Chr.)}

Unser Versuch, die spärlichen Nachrichten über Hypsikrateia zu ergänzen und in einen dichteren Traditionszusammenhang zu stellen, können wir mit einer weiteren Überlegung abrunden. Es ist mittlerweile klar geworden, dass die nächtliche Flucht des Mithradates ein wichtiges Thema der historischen Überlieferung war. Selbst in der knappen Inhaltsangabe zu Livius, Buch 101 sticht sie noch prominent hervor: Cn. Pompeius Mithridaten nocturno proelio victum coegit Bosporum profugere (Per. 101). ${ }^{32}$ Das ist kein Wunder, denn diese Flucht war das Ergebnis der letzten großen Feldschlacht, die Mithradates gegen die Römer schlug. Dieses Ereignis war so wichtig und ein so bedeutender Erfolg des Pompeius, dass dieser es auf Bildtafeln seines im Jahre 61 in Rom durchgeführten Triumphzuges darstellen ließ. Überaus eindrucksvoll, ja einmalig war die Zahl der besiegten Völker und der unterworfenen Gebiete, über die Pompeius triumphieren konnte. Über zwei Tage (28. und 29. September 61) erstreckte sich das Schauspiel in den Straßen Roms. Nach dem Bericht des Historikers Appian, Verfasser einer Römischen Geschichte im 2. Jh. n. Chr., zogen Tausende von Kriegsgefangenen und Hunderte Offiziere und Angehörige besiegter Königshäuser an den Zuschauern des Triumphzuges vorüber. Mithradates, der sich durch Freitod einer möglichen Gefangennahme entzogen hatte (dazu weiter unten), wurde bei diesem Zug auf Schaubildern dargestellt: seine nächtliche Schlacht, seine Niederlage, seine Flucht und sein Tod (App. Mithr. 574f.): „(574) Es wurden Schaubilder der Nichtanwesenden vorüber getragen, des

\footnotetext{
31 Die von Eutrop genannte Zahl von drei Begleitern passt zu der entsprechenden Angabe von Plut. Pomp. 32, 14. Vgl. in diesem Sinne schon Ballesteros Pastor 2011, S. 116.

32 Vgl. auch die auf Livius zurückgehende Darstellung des Cassius Dio 36, $48 \mathrm{f}$.
} 
Tigranes und des Mithridates, als Kämpfende, als Besiegte und als Fliehende. Dargestellt waren auch die Belagerung des Mithridates und die Nacht, als er floh, sowie Sinope. (575) Am Schluss wurde auch gezeigt, wie er starb; auch die jungen Frauen, die sich entschlossen hatten, gemeinsam mit ihm zu sterben, waren neben ihm im Bild dargestellt. Bilder seiner vor ihm verstorbenen Söhne und Töchter waren ebenfalls vertreten sowie Bildnisse von Barbarengöttern und Nationalkostüme." (Übers.: H. Heinen)

Wir dürfen sicher sein, dass das in diesem Appian-Passus erwähnte Schaubild seiner Flucht den geschlagenen und verlassenen König in Begleitung Hypsikrateias darstellte. Man sollte nicht so weit gehen wie Paul Goukowsky in seinem Kommentar des Mithradates-Buches Appians und die auf dem Triumphzug mitgeführten Bildtafeln für angebliche Fehler der schriftlichen Überlieferung verantwortlich machen. ${ }^{33}$ Eher ist der umgekehrte Vorgang anzunehmen: Die großen Erfolge des Pompeius wurden bereits vor seinem erst 61 v. Chr. stattfindenden Triumphzug nicht nur mündlich verbreitet, sondern auch schon vor diesem Zeitpunkt schriftlich gefeiert, vor allem in einem Werk des dem Pompeius nahestehenden Theophanes von Mytilene. ${ }^{34}$ Derartige Informationen und vor allem die offiziellen Berichte des Pompeius dürften die Auswahl und die Inhalte der Szenen bestimmt haben, die wenig später in den historischen Schlachtdarstellungen des römischen Triumphzuges gezeigt wurden. ${ }^{35}$

\section{Das Ende des Mithradates und das Schicksal seiner Familie}

Vorhin schon war davon die Rede, dass Hypsikrateia in den letzten schweren Kämpfen des Mithradates nach seiner Flucht ins Bosporanische Reich nicht erwähnt wird, obwohl Appian in seiner Darstellung dieser dramatischen Ereignisse ziemlich detailliert über Treue und Verrat in der königlichen Familie, über die Opfer auf beiden Seiten und über den gegen Mithradates gerichteten Aufstand der Stadt Phanagoreia, dem Fundort des aus dem Küstengewässer gehobenen Grabdenkmals Hypsikrateias, berich-

33 Appien 2003, S. 251 Anm. 1096 : „Il n’est pas impossible que le récit de la bataille nocturne (dont Appien n’a pas fait état ...) ait été élaboré à partir de ce tableau par des historiens peu soucieux de la vérité." - Zur Überlieferung über die Schlacht und Flucht des Mithradates vgl. Heftner 1995, S. 224-233, mit guten Beobachtungen.

34 Zum Kontext der Veröffentlichung des Theophanes vgl. Heftner 1995, S. 53-59, und Engels 1999, S. 171f.

35 Östenberg 2009, S. 246-261, zufolge bestanden viele dieser Darstellungen aus belebten Bildern. Auch Flucht und Tod des Mithradates und seiner Begleitung seien wahrscheinlich durch menschliche Darsteller inszeniert worden, „who performed simple movements against painted settings" (S. 253). 
tet. Auch unter den gefangenen und auf dem Triumphzug des Pompeius vorgeführten Familienmitgliedern des Mithradates wird Hypsikrateia nicht genannt. Vieles scheint dafür zu sprechen, dass sie den Tod in den Wirren der letzten Kämpfe des Mithradates gefunden hat, ohne dass ihr Ende eine Spur in den auf uns gekommenen literarischen Quellen hinterlassen hätte. In Appians Darstellung der mithradatischen Kriege hätte man am ehesten eine Nachricht über ihren Tod erwartet, doch seine Aufmerksamkeit gilt vor allem den Söhnen und Töchtern des Königs. So bleiben denn wohl die genauen Umstände von Hypsikrateias Ende und Bestattung im Dunkeln. Doch die Wahl der männlichen Namensform Hypsikrates auf ihrem Grabstein hält für immer fest, dass Hypsikrateia unter den Frauen des Königs bis zuletzt eine besondere, männliche und kriegerische, Rolle gespielt hat.

Wenngleich es die Quellenlage nicht ermöglicht, die näheren Umstände von Hypsikrateias Tod aufzuklären, so sind wir doch über die letzten Tage des Mithradates und das dramatische Ende seiner Herrschaft gut informiert. Die antiken Autoren, allen voran Appian in seinem MithradatesBuch, bieten einen relativ detaillierten Überblick über den Untergang des Königs und liefern zudem zahlreiche Details, aus denen sich ein Szenario rekonstruieren lässt, in dem auch Hypsikrateia den Tod gefunden haben könnte. ${ }^{36}$ Mir geht es im Folgenden allerdings nicht primär um die kritische Rekonstruktion der politischen und militärischen Ereignisse und auch nicht um den Versuch, für die Errichtung des Grabdenkmals der Hypsikrateia einen möglichst plausiblen Kontext zu erschließen. Zu diesen wichtigen Fragen gibt es bereits mehrere Untersuchungen. ${ }^{37}$ Der nachstehende Überblick verfolgt vielmehr den Zweck, die Schlussphase der Herrschaft des Mithradates zu skizzieren, denn sie ist, wie gesagt, relativ gut dokumentiert und hätte deshalb eine Nennung Hypsikrateias erwarten lassen. Vor dieser nun zu skizzierenden Folie sind der von Valerius Maximus gewählte Ausschnitt aus dem Schicksal Hypsikrateias und seine Akzentuierung ihrer Gestalt umso bemerkenswerter.

Nach der Niederlage des Jahres 65 in der Entscheidungsschlacht gegen Pompeius hatte sich Mithradates aus Kleinasien über die Ostküste des Schwarzen Meeres in das Bosporanische Reich zurückgezogen. Dort herrschte bis dahin sein Sohn Machares, der sich jedoch schon früher mit den Römern verständigt hatte. Das wurde ihm nun zum Verhängnis. Ange-

\footnotetext{
App. Mithr. 502-550.

37 Vgl. P. Bernard apud Bongard-Levine u.a. 2006, S. 279-288; Kuznecov 2007, S. 241-143; Bowersock 2008, S. 600 f.; Gabelko 2009; neue numismatische Daten zum Aufstand Phanagoreias: Abramzon/Kuznecov 2011. S. zum Gesamtkontext auch Reinach 1895, S. 375-410, und Ballesteros Pastor 1996, S. 277-282.
} 
sichts des raschen Vormarsches seines Vaters, der inzwischen wieder Truppen um sich gesammelt hatte, versuchte Machares zu fliehen. Dabei verlor er das Leben, sei es durch Selbstmord aus Angst vor der drohenden Bestrafung durch seinen Vater, sei es auf Befehl des Letzteren. Die politischen Ratgeber, die er einst seinem Sohn zur Seite gestellt hatte, ließ Mithradates hinrichten. ${ }^{38}$ Allein schon diese Episode zeigt, mit welch ungeheurer Verbitterung und Brutalität der König auch gegen seine eigenen Familienangehörigen und Freunde vorging, wenn er Verrat witterte. Nach dem Tode des Machares übte Mithradates nun selber die Herrschaft über das Bosporanische Reich aus, nahm Verbindung zu den benachbarten Skythen auf und versuchte, ein neues schlagkräftiges Heer zwecks Fortsetzung des Krieges aufzustellen. ${ }^{39}$ Diese mit großer Energie und Härte von Pantikapaion aus betriebenen Maßnahmen lösten im Jahre 63 in Phanagoreia, auf der asiatischen Seite des Kimmerischen Bosporos, einen Aufstand unter Führung eines gewissen Kastor aus. Der Burghügel von Phanagoreia wurde belagert, denn auf ihm befanden sich einige Söhne und Töchter des Mithradates. Die meisten von ihnen gaben auf und ließen sich gefangen nehmen. Nicht so jedoch seine Tochter Kleopatra, die den Widerstand fortsetzte und schließlich durch eine Flottenoperation des Königs aus ihrer prekären Lage befreit wurde. Doch der Aufstand griff weiter um sich. Die wichtigsten Städte und befestigten Plätze des Bosporanischen Reiches schlossen sich der Erhebung an. Mithradates konnte seiner eigenen Truppen nicht mehr sicher sein. Eine starke Reiterabteilung erhielt den Auftrag, einige Töchter des Königs zu skythischen Fürsten zu geleiten. Ihre Verheiratung sollte Mithradates die politische und militärische Unterstützung der benachbarten Skythen sichern. Doch die Reiter töteten die Eunuchen, die zum begleitenden Hofstaat der Töchter gehörten, und lieferten die jungen Frauen an die Römer aus. ${ }^{40}$

In der Stimmung dieses um sich greifenden Abfalls erhob sich ein weiterer Sohn, Pharnakes, gegen den König und gewann rasch Zulauf bei den Truppen..$^{41}$ Mithradates mussten nun gegen seinen eigenen Sohn um seinen Thron und sein Leben kämpfen. Doch aller Widerstand war umsonst. Während Pharnakes sich unter tumultartigen Umständen zum König krönen ließ, bereitete Mithradates seinen Selbstmord vor. Seine bei ihm verbliebenen Töchter Mithridatis und Nyssa bestanden darauf, vor ihrem Va-

\footnotetext{
38 App. Mithr. 476.

39 App. Mithr. 508-510.

40 App. Mithr. 516.

41 App. Mithr. 523ff.
} 
ter Gift zu nehmen. ${ }^{42}$ Die beiden starben vor den Augen des Mithradates, doch bei ihm selber verfehlte das Gift eine schnelle Wirkung. Durch die regelmäßige Einnahme von Gegenmitteln hatte der König sich in der Vergangenheit gegen Giftanschläge zu schützen versucht und konnte nun nicht sterben. ${ }^{43}$ Schließlich ließ er sich durch einen seiner Soldaten töten, um seine Gefangennahme zu verhindern. ${ }^{44}$ Soweit der Bericht Appians. Dreiunddreißig Jahre später sollte Kleopatra, die letzte Königin Ägyptens, ihrerseits den Freitod wählen, um der Schmach zu entgehen, von Octavian, dem späteren Kaiser Augustus, der Bevölkerung Roms lebend auf dem Triumphzug vorgeführt zu werden.

Bereits seinerzeit, bei seiner Flucht nach der entscheidenden Niederlage gegen Pompeius, hatte Mithradates Gift an seine Freunde verteilt, damit keiner von ihnen gegen seinen Willen in die Hände der Feinde geriete. ${ }^{45}$ Vermutlich hatte auch Hypsikrateia damals diese tödliche und wohl doch willkommene Gabe ihres Geliebten erhalten. Doch sie brauchte das Gift jedenfalls damals nicht einzunehmen, denn, wie wir wissen, sollte ihr und Mithradates die Flucht zur Nordküste des Schwarzen Meeres gelingen.

Soweit der dramatische Ablauf der letzten Tage des Königs, wie ihn der griechische Historiker Appian, unsere ausführlichste Quelle, darstellt. Den auf Livius fußenden kaiserzeitlichen Epitomatoren verdanken wir den bei Appian fehlenden Hinweis, dass damals auch die Frauen und Konkubinen von Mithradates Gift entgegennahmen und starben. ${ }^{46}$ Leicht könnte man vermuten, dass Hypsikrateia zum Kreis dieser todgeweihten Frauen gehörte. Doch ihr in Phanagoreia entdecktes Denkmal weist eher darauf hin, dass sie im Gegensatz zu den vorhin genannten Frauen nicht auf der Königsburg von Pantikapaion starb, sondern wohl eher in den Kämpfen um Phanagoreia das Leben verlor. Aber sicher ist das nicht.

Bedenkt man die Tragödien der Frauen, die sich beim Untergang des Mithradates abgespielt haben, so wundert man sich, dass Valerius Maximus bei seiner Schilderung exemplarischer Gattenliebe nicht etwa gesagt hat, dass Hypsikrateia aus Liebe zu Mithradates mit ihm in den Tod gegangen sei oder in den Kämpfen der Endphase seines Reiches ihr Leben verloren habe. Nein, für Valerius Maximus ist der Beweis ihrer Liebe für den König ihr ausdauernder, anstrengender Dienst an seiner Seite und ihre

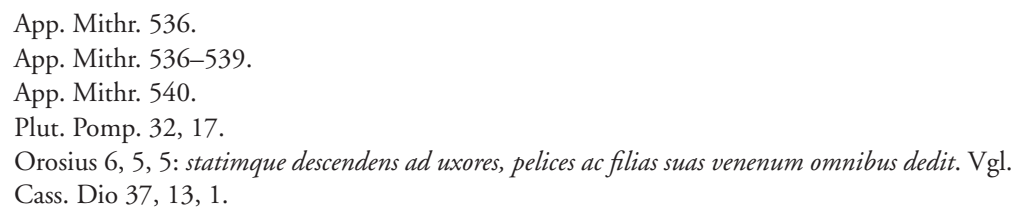


Treue auch in der Niederlage. Das höchste Opfer Hypsikrateias, von dem Valerius Maximus zu berichten weiß, ist nicht ihr Leben, sondern die Opferung ihres Haares, das sich die junge Frau abschneidet, um in männlicher Aufmachung den König als Page begleiten zu können. Nicht ihr Tod, sondern ihre Travestie aus Liebe ist sein Thema. ${ }^{47}$

\section{Hypsikrateia, Gustav Adolfs Page und eine weitere Travestie aus Liebe: die Figur der Plotina bei Apuleius}

Als treue, liebende und verwegene Begleiterin des Mithradates hat uns Hypsikrateia eingangs an Gustav Adolfs Pagen Leubelfing erinnert. Die Parallele zwischen den beiden Frauen lässt sich bis zum Ende ihrer Lebenskurve und über ihren Tod hinaus verfolgen. Denn auch der Phantasiegestalt Gustl Leubelfings wurde in Conrad Ferdinand Meyers Novelle ein Grabstein gesetzt, der nach dem Willen des strengen protestantischen Pfarrers Magister Todänus die weibliche Identität des Pagen verschweigen sollte, um das Andenken und die Reputation Gustav Adolfs zu schützen. ${ }^{48}$ Der sterbenden Gustl versichert der Pfarrer:

„Ihr seid der Page August Leubelfing, ehelicher Sohn des nürembergischen Patriziers und Handelsherrn Arbogast Leubelfing, geboren den und den, Todes verblichen den siebenten November Eintausend sechshundert zweiunddreißig an seinen Tages vorher in der Schlacht bei Lützen empfangenen Wunden, pugnans cum rege Gustavo Adolpho.

,Fortiter pugnans!' ergänzte der Cornett begeistert.

,So will ich auf Euren Grabstein setzen! Jetzt aber machet Euern Frieden mit Gott! Euer Stündlein ist gekommen.’ Der Magister sagte das nicht ohne Härte, denn er konnte seinen Unmut gegen das abenteuerliche Kind, das den Ruf seines Helden gefährdet hatte, nicht verwinden, ob es schon in den letzten Zügen lag. “ ${ }^{49}$

Kehren wir abschließend noch einmal zur Antike zurück und werfen wenigstens einen raschen Blick auf eine Figur, die gewisse parallele Züge mit Hypsikrateia aufweist. Es handelt sich um Plotina im 7. Buch der Metamorphosen bzw. des Goldenen Esels des Apuleius (geb. 125 n. Chr.). Der Kontext ist ein geläufiges Szenario antiker Romane. Die fiktive Handlung spielt in Griechenland im 2. Jahrhundert n. Chr.: Charite, die Braut des

47 Interessanterweise handelt auch das dritte Exemplum, das Valerius Maximus an dasjenige Hypsikrateias anschließt, von einer Travestie, diesmal mit Täuschungsabsicht: Die Minyerinnen in Sparta legen die Kleidung ihrer Männer an und retten so deren Leben (Val. Max. 4, 6 ext. 3).

48 Zu Meyers Umgang mit dem historischen Stoff vgl. Osborne 2000.

49 Meyer 1959, S. 212 f. 
vornehmen Tlepolemos, ist in die Hände von Räubern gefallen. Um sie zu befreien, gibt Tlepolemos sich selber als der Räuberhauptmann Haemus aus Thrakien aus und gewinnt auf diese Weise das Vertrauen der Entführer. Als Beweis seiner Räuberkarriere tischt Tlepolemos der Bande die erfundene Geschichte eines zunächst erfolgreichen Überfalls auf, der jedoch durch die Intervention kaiserlicher Soldaten in einer Katastrophe endet. Mir geht es hier vor allem um die Figur der Plotina, die ihren hochgestellten Mann entsagungsvoll in die Verbannung begleitet (Apul. 7, 6, 2f.).

Sed uxor eius Plotina, quaedam rarae fidei atque singularis pudicitiae femina, quae decimo partus stipendio viri familiam fundaverat, spretis atque contemptis urbicae luxuriae deliciis fugientis comes et infortunii socia, tonso capillo, in masculinam faciem reformato habitu, pretiosissimis monilium et auro monetali zonis refertis incincta inter ipsas custodientium militum manus et gladios nudos intrepida cunctorum periculorum particeps et pro mariti salute pervigilem curam sustinens, aerumnas adsiduas ingenio masculo sustinebat.

„Aber seine Frau Plotina, ein Weib von seltener Treue und besonderer Sittsamkeit, die ihrem Mann mit der Leistung von zehn Geburten eine Familie gegründet hatte, wollte nun von den Genüssen des städtischen Luxus ganz und gar nichts mehr wissen, und als Begleiterin des Verbannten und Gefährtin seines Unglücks, das Haar geschoren, die Kleidung nach männlicher Art umgewandelt, gegürtet mit einem Gürtel, der angefüllt war mit ihrem kostbarstem Schmuck und gemünztem Gold, so teilte sie mitten zwischen den Scharen der bewachenden Soldaten und den bloßen Schwertern furchtlos alle Gefahren, und indem sie die stets wachsame Fürsorge für das Wohl ihres Mannes auf sich nahm, ertrug sie die andauernden Mühseligkeiten mit einem männlichen Geiste." (Übersetzung: R. Helm) $)^{50}$

Es würde den vorgegebenen Rahmen sprengen, wenn ich hier die ganze Geschichte des Tlepolemos wiedergeben wollte. Doch nur noch so viel: Er entkommt dem Gemetzel, indem er sich als Frau verkleidet (7, 8, 1: sumpta veste muliebri).

Allein schon diese Sequenz bietet eine verwirrende Reihe von Masken und Travestien: Tlepolemos tritt als Räuber Haemus auf, entkommt in Frauenkleidern nach dem Überfall auf eine übrigens fiktive Reisegruppe, deren auffälligste Figur Plotina, eine Frau in Männerkleidung, darstellt.

Plotina und Hypsikrateia bilden keine perfekte Parallele: Plotina ist Mutter von zehn Kindern, Hypsikrateia eine wahrscheinlich sehr junge Gefährtin des Mithradates. Kinder aus dieser Verbindung sind nicht bekannt und hat es wohl auch nicht gegeben. Aber die Parallelen zwischen den bei-

50 Apuleius, Metamorphosen oder Der Goldene Esel. Lat. und dt. von R. Helm, Berlin 1956, S. 196f. S. zu Apul. 7, 6 den Kommentar von Hijmans 1981, S. 117-125, sowie MüllerReineke 2008, spez. zu den möglichen Anspielungen auf Trajans Gattin Plotina. Den Hinweis auf den Aufsatz von Müller-Reineke verdanke ich Judith Hindermann/Basel. 
den Figuren sind demgegenüber deutlich und überraschend: Beide begleiten ihren Partner in die Verbannung, beide legen männliche Kleidung an und lassen sich die Haare kurz schneiden, beide zeigen ungewöhnlichen Mut, beide harren in großer Not und Bedrängnis an der Seite ihres Partners aus und beide beabsichtigen mit ihrer Travestie keine Täuschung ihres Geliebten und ihrer Umgebung. Doch nicht so sehr die eheliche Treue verleiht den beiden Frauen ihr besonderes Profil, denn Treue wurde auch an anderen griechischen und römischen Frauen gerühmt. Nein, die besondere Gemeinschaft Plotinas und Hypsikrateias besteht darin, dass sie sich äußerlich gewissermaßen in Männer verwandeln und männlichen Mut beweisen, wobei der Fall Hypsikrateias noch eine Steigerung dadurch erfährt, dass Mithradates seiner Gefährtin einen männlichen Namen verleiht. Die höchste Steigerung erreicht jedoch, wenn wir die vorhin besprochenen Figuren Revue passieren lassen, Gustav Adolfs Page Leubelfing, denn dieser bewahrt bis in den Tod das sorgfältig gehütete Geheimnis seines wahren weiblichen Geschlechts und hütet es besonders sorgfältig vor dem von ihr geliebten und verehrten Schwedenkönig, dem strengen und viel älteren Gustav Adolf.

\section{Schlussbetrachtungen}

Die Travestien Hypsikrateias, Plotinas und Gustl Leubelfings vollzogen sich auf unterschiedliche Weise und in verschiedenen kulturellen Konstellationen. Im Unterschied zu den fiktiven Inszenierungen Plotinas und der Gustl Leubelfing ist das Schicksal Hypsikrateias konkrete historische Realität und bis zu einem gewissen Grade auf einem echten Denkmal überprüfbar. Doch jenseits aller Unterschiede ist diesen Frauen gemeinsam, dass sie aus Liebe zu einem Mann einen Teil ihrer weiblichen Identität aufgeben oder verbergen. Sie repräsentieren nicht nur den Typus der in einer Männerrolle auftretenden Begleiterin oder Kriegerin, wie sie in Liedern und Geschichten vielfach geschildert wird, sondern sind letztlich Beispiele für die verwandelnde Kraft der Liebe. Metamorphose aus Liebe, nicht jedoch der Rückgriff auf den Amazonentypus ist der entscheidende Aspekt, unter dem Valerius Maximus in dem eingangs zitierten Text die Gestalt der Hypsikrateia dargestellt und konstruiert hat.

Giovanni Boccaccio hatte in seiner Schrift De mulieribus claris noch verkündet, dass der Name Hypsikrateia nie in Vergessenheit geraten werde: nomen eius monimentis venerandarum literarum ad nos usque et in perpetuum fama celebri vivet - „Ihr Name wird dank verehrungswürdiger Schriftdenk- 
mäler bis in unsere Zeit und in Ewigkeit glorreich weiterleben... ".51 Doch der Benutzer von Pauly-Wissowas Realencyclopädie der classischen Altertumswissenschaft muss erstaunt feststellen, dass das Lemma „Hypsikrateia“ trotz der bewundernswerten Fülle dieses Schatzhauses fehlt. Den Eintrag „Hypsikrateia" sucht man ebenfalls vergeblich im Kleinen Pauly und im Neuen Pauly. Obwohl Hunderte von Handschriften des Valerius Maximus erhalten sind und die Gestalt Hypsikrateias in der Zeit des Humanismus und der Renaissance sehr bekannt war, ist sie später aus dem Blick geraten. Ihre Wiederentdeckung verdanken wir den Ausgräbern von Phanagoreia.

\section{Bibliographie}

Abramzon, M.G./Kuznecov, V.D.: Novye dannye o vosstanii 63 g. do n.ë. v Fanagorii (Neue Daten zum Aufstand des Jahres 63 v.u.Z. in Phanagoreia) (russ.). In: VDI 2011 (2) S. 64-95

Altenburger, R.: The Sword or the Needle. The Female Knight-errant (xia) in Traditional Chinese Narrative. Bern u.a. 2009

Appien. Histoire Romaine. Tome VII. Livre XII. La Guerre de Mithridate. Texte établi et traduit par Paul Goukowsky, Paris 2003

Ballesteros Pastor, L.: Mitrídates Eupátor, rey del Ponto. Granada 1996

Ballesteros Pastor, L.: La leyenda de las Amazonas en la historia de Mitrídates Eupátor. In: J.M. Cortes Copete u.a. (Hrsg.): X $\alpha \iota \mathrm{P} \rho \varepsilon$. Actas de la II Reunión de Historiadores del Mundo Griego Antiguo. Homenaje al Profesor Fernado Gascó. Sevilla 1997, S. 241247

Ballesteros Pastor, L.: El relato sobre Hipsicratea (Plut., Pomp.32.7-8) y la imagen de Mitrídates en Plutarco. In: J.M. Candau Morón/F.J. González Ponce/A.L. Chávez Reino (Hrsg.): Plutarco Transmisor. Actas del X Simposio Internacional de la Asociación española de Plutarquistas (Sevilla 2009). Sevilla 2011, S. 113-122

Bang, C. K.: Maske und Gesicht in den Werken Conrad Ferdinand Meyers. Baltimore 1940

Bohm, C.: Imitatio Alexandri im Hellenismus. Untersuchungen zum politischen Nachwirken Alexanders des Großen in hoch- und späthellenistischen Monarchien. München 1989

Bongard-Levine, G., Kochelenko, G. \& Kouznetsov, V.: Fouillles de Phanagorie: nouveaux documents archéologiques et épigraphiques du Bosphore. In: Académie des Inscriptions \& Belles-Lettres, Comptes Rendus. Paris 2006, 255-292

Bosch, C.: Die Quellen des Valerius Maximus. Ein Beitrag zur Erforschung der Litteratur der historischen Exempla. Stuttgart 1929

Bowersock, G.W.: In Search of Strabo, with Some New Light on Mithridates Eupator and His Concubine. In: Journal of Roman Archaeology 21 (2008) 598-601

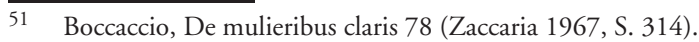


Canali De Rossi, F.: Rezension zu A. Coşkun (Hrsg.): Freundschaft und Gefolgschaft in den auswärtigen Beziehungen der Römer (2. Jahrhundert v. Chr. - 1. Jahrhundert n. Chr.). Frankfurt am Main u.a. 2008. In: BMCR 2009.05.22

Engels, J.: Augusteische Oikumenegeographie und Universalhistorie im Werk Strabons von Amaseia. Stuttgart 1999

Fornasier, J.: Amazonen. Frauen, Kämpferinnen und Stadtgründerinnen. Mainz 2007

Gabelko, O.L.: Istoriko-ëpigrafièeskij kommentarij k nadgrobnoj nadpisi Gipsikratii (Historisch-epigraphischer Kommentar zur Grabinschrift der Hypsikrateia) (russ.). In: Voprosy ëpigrafiki 3 (2009) S. 188-201

Gardiner-Garden, J. R.: Fourth Century Conceptions of Maiotian Ethnography. In: Historia 35 (1986) S. 192-225

Heftner, H.: Plutarch und der Aufstieg des Pompeius. Ein historischer Kommentar zu Plutarchs Pompeiusvita. Teil I: Kap. 1-45. Frankfurt/Main 1995

Heinen, H.: Romfreunde und Kaiserpriester am Kimmerischen Bosporos. Zu neuen Inschriften aus Phanagoreia, in: A. Coşkun (Hrsg.): Freundschaft und Gefolgschaft in den auswärtigen Beziehungen der Römer (2. Jahrhundert v. Chr. - 1. Jahrhundert n. Chr.). Frankfurt am Main u.a. 2008, S. 189-208

Hijmans, B.L. u.a: Apuleius Madaurensis. Metamorphoses, Books VI 25-32 and VII. Text, Introduction and Commentary (Groningen Commentaries on Apuleius). Groningen 1981

Historisches Museum der Pfalz Speyer (Hrsg.). Amazonen. Geheimnisvolle Kriegerinnen. Speyer 2010 (Kataloghandbuch)

Klotz, A.: Studien zu Valerius Maximus und den Exempla. München 1942

Kuznecov, V. D.: Novye nadpisi iz Fanagorii (Neue Inschriften aus Phanagoreia) (russ.): In: Vestnik drevnej istorii 2006 (1) S. 155-172

Kuznecov, V. D.: Novye nadpisi iz Fanagorii (Neue Inschriften aus Phanagoreia) (russ.): In: Vestnik drevnej istorii 2007 (1) S. 227 - 243

Kuznetsov, V., Povalahev, N. (Hrsg.): Phanagoreia.Die vergessene Metropole am Schwarzen Meer. Eine Expedition berichtet. Göttingen 2009

Mayor, A.: Pontisches Gift. Die Legende von Mithridates, Roms größtem Feind, aus dem Engl. übersetzt von H. Dierlamm und N. Juraschitz. Stuttgart 2011 (engl. Original: The Poison King. The Life and Legend of Mithradates, Rome's Deadliest Enemy. Princeton, Oxford 2010)

Meyer, C. F.: Novellen I (Conrad Ferdinand Meyer: Sämtliche Werke, historisch-kritische Ausgabe besorgt von H. Zeller und A. Zäch, Bd. 11). Bern 1959

Montero, S.: Mujeres extranjeras en la obra de Valerio Máximo. In: G. Bravo Castañeda/ R. González Salinero (Hrsg.): Extranjeras en el mundo romano (Gerión-Anejos VIII). Madrid 2004, S. 45-56

Müller-Reineke, H.: rarae fidei atque singularis pudicitiae femina - The Figure of Plotina in Apuleius' Novel (Metamorphoses 7. 6-7). In: Mnemosyne 61 (2008) S. 619-633

Östenberg, I.: Staging the World. Spoils, Captives, and Representations in the Roman Triumphal Procession. Oxford 2009

Orosius, s. Paulus Orosius 
Osborne, J.: Zum Geschichtsverständnis Conrad Ferdinand Meyers am Beispiel von Gustav Adolfs Page. In: R. Zeller (Hrsg.): Conrad Ferdinand Meyer im Kontext. Beiträge des Kilchberger Kolloquiums. Heidelberg 2000, S. 189-204

Paulus Orosius. Die antike Weltgeschichte in christlicher Sicht. Buch V-VII, übersetzt und erläutert von A. Lippold, Zürich, München 1986

Plutarch. Große Griechen und Römer, eingeleitet und übersetzt von K. Ziegler. Bd. III. Zürich, Stuttgart 1955

Reinach, Th.: Mithradates Eupator, König von Pontus, mit Berichtigungen und Nachträgen des Verfassers ins Deutsche übertragen von A. Goetz. Leipzig 1895

Saprykin, S. Ju.: Bosporskoe carstvo na rubeže dvuch ëpoch (Das Bosporanische Reich an der Scheide zweier Epochen). (russ.). Moskau 2002

Savostina, E. (Hrsg.): Bosporan Battle Relief (Amazonomachia?)/Bosporskij rel'ef so scenoj sraženija (Amazonomachija?). Moskau, St. Petersburg 2001

Seemann, E.: Die Gestalt des kriegerischen Mädchens in den europäischen Volksballaden. In: Rheinisches Jahrbuch für Volkskunde 9 (1959) S. 192-212

Smirnov, K.F.: Une „amazone“ du IV ${ }^{e}$ siècle avant n.è. sur le territoire du Don. In: Dialogues d'histoire ancienne 8 (1982) S. 121-141

Themann-Steinke, A.: Valerius Maximus. Ein Kommentar zum Zweiten Buch der Facta et Dicta memorabilia. Trier 2008

Thurn, N.: Der Aufbau der Exemplasammlung des Valerius Maximus. In: Hermes 129 (2001) S. 79-94

Valère Maxime. Faits et Dits Mémorables. Tome I, Livres I-III. Texte établi et traduit par Robert Combès. Paris 1995

Wiesmann, L.: Conrad Ferdinand Meyer. Der Dichter des Todes und der Maske. Bern 1958

Zaccaria, V. (Hrsg.): De Mulieribus Claris (Tutte le opere di Giovanni Boccaccio, a cura di V. Branca, vol. X). Mailand 1967

\section{Abkürzungen}

Bull. ép. = Bulletin épigraphique (in Revue des Etudes Grecques)

$\mathrm{RE}=$ Pauly-Wissowas Realencyclopädie der classischen Altertumswissenschaft

SEG $=$ Supplementum Epigraphicum Graecum

VDI = Vestnik drevnej istorii

\section{Abbildungsnachweis}

Abbildung 1: Hupe, J. (Hrsg.): Der Achilleus-Kult im nördlichen Schwarzmeerraum vom Beginn der griechischen Kolonisation bis in die römische Kaiserzeit. Beiträge zur Akkulturationsforschung. Rahden/Westf. 2006, Taf. 1; Entwurf J. Hupe, graphische Bearbeitung D. Engels (Atelier AAK 1-Design) 
Abbildung 2: Kuznecov, V.D.: Novye nadpisi iz Fanagorii (Neue Inschriften aus Phanagoreia) (russ.): In: Vestnik drevnej istorii 2007 (1) Abbildung 6 auf Tafel nach S. 128.

Abbildung 3: Kuznecov: Ebd., S. 240, Abbildung 9, 1

Für die Bereitstellung der Abbildungsvorlagen danke ich Herrn Dr. Joachim Hupe (Karte Abbildung 1) sowie den Herren Professor Dr. Askold I. Ivantchik und Dr. V.D. Kuznecov (Abbildung 2 und 3). 